\title{
Bayesian hypothesis testing for the origin of the methane in Enceladus' plume: biomethanation versus abiotic hydrothermal chemistry.
}

\author{
ANTONIN AFFHOLDER ${ }^{1,2}$, FRANCOIS GUYOT ${ }^{3}$, BORIS \\ SAUTEREY $^{1}$, STÉPHANE MAZEVET ${ }^{2}$ AND RÉGIS \\ FERRIÈRE $^{1,4}$ \\ ${ }^{1}$ Institut de Biologie de l'École Normale Supérieure \\ ${ }^{2}$ Observatoire de Paris \\ ${ }^{3}$ Institut de Minéralogie, Physique des Matériaux et \\ Cosmochimie, CNRS UMR 7590 \\ ${ }^{4}$ Department of Ecology and Evolutionary Biology \\ Presenting Author: antonin.affholder@bio.ens.psl.eu
}

The Saturnian satellite Enceladus has attracted much interest in the search for habitable environments in the Solar system $[1,2]$. NASA's Cassini probe revealed the existence of a global ocean of circulating water underneath Enceladus' icy surface $[3,4,5,6]$. The onboard instrument Ion and Neutral Mass Spectrometer (INMS) performed in situ chemical analysis of oceanic material composing large plumes which supports the existence of Earth-like alkaline hydrothermal vents at Enceladus' seafloor [1]. Earth's alkaline vents harbor complex ecosystems rich in methanogenic archaea [7], thus raising the possibility that similar organisms are present in Enceladus' putative vents. Here, we use a thermodynamically-grounded model of a methanogenic ecosystem coupled to a model of ocean circulation in order to evaluate the likelihood that the observed ejection rates of volatiles in the plume are caused by biomethanation rather than in serpentinization-related chemistry. We find that (i) the observed escape rate of hydrogen is consistent with favorable conditions for methanogens at the bottom of Enceladus' ocean, (ii) the escape rate of methane is needed to evaluate the likelihood of methanogens being actually present and (iii) methane escape rate expected from a population of methanogens performs much better than serpentinization-related chemistry at explaining Cassini's observations. We conclude that methanogens are a likelier explanation of Enceladus' plume composition than serpentinization alone, thus stressing the importance for future space missions to investigate and identify potential alternative abiotic sources of methane.

[1] Waite J. H. et al., (2017), Science, 356, 155-159. [2] Taubner R. S. et al., (2018), Nature communications, 9, 1-11. [3] Waite Jr J. H et al., (2009), Nature, 460, 487-490. [4] Postberg F et al., (2009), Nature, 459, 1098-1101. [5] Travis B. J. \& Schubert G., (2015), Icarus, 250, 32-42. [6] Choblet G et al., (2017), Nature Astronomy, 1, 841-847. [7] Schrenk M. O et al., (2004), Environmental Microbiology, 6, 1086-1095. 